\title{
Painless pink papules with central porcelain-white scars
}

A

61-year-old woman presented with a 2-year history of asymptomatic, erythematous to pink papules with central porcelainwhite atrophic scars and a telangiectactic rim (Figure 1). A progressive number of lesions had developed on her abdomen, back and thighs. Skin histology showed epidermal atrophy, a vacuolar interface reaction and a wedge-shaped area of collagen degeneration and papillary dermal sclerosis. The results of our investigations are summarized in Table 1.

\section{What is your diagnosis?}

a. Lichen sclerosus

b. Guttate morphea

c. Systemic lupus erythematosus

d. Malignant atrophic papulosis

e. Atrophie blanche

See page 1172 for the diagnosis.

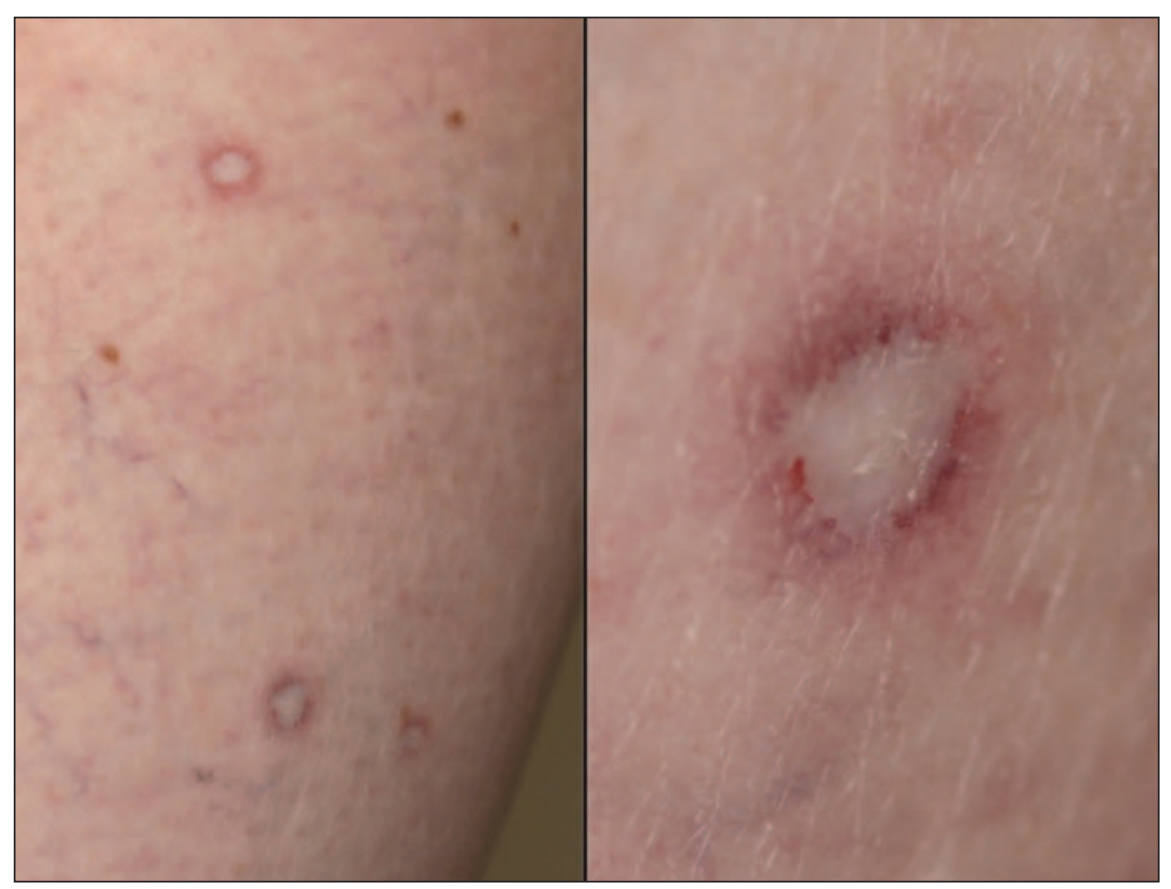

Figure 1: Asymptomatic, erythematous to pink papules with central porcelain-white atrophic scars and telangiectactic rims.

\begin{tabular}{ll}
\hline Table 1: Summary of investigations & \\
\hline Investigation & \multicolumn{1}{c}{ Result } \\
\hline $\begin{array}{l}\text { Complete blood count with differential, hepatorenal } \\
\text { and coagulation profiles }\end{array}$ & Within normal limits \\
$\begin{array}{l}\text { Rheumatoid factor, antinuclear antibody, } \\
\text { antiphospholipid antibody }\end{array}$ & Negative \\
$\begin{array}{l}\text { Urinalysis } \\
\text { Guaiac test for occult blood in stool specimen } \\
\text { Chest radiography }\end{array}$ & Within normal limits \\
Magnetic resonance imaging of head & Negative \\
Endoscopy and colonoscopy & Within normal limits \\
\hline
\end{tabular}




\section{WHAT IS YOUR CALL?}

\section{Discussion}

Based on the clinical and pathological findings, the answer is (d) malignant atrophic papulosis (also known as Degos disease). This condition is a rare but potentially fatal occlusive arteriopathy of small to medium-sized vessels. Patients may have a benign variant of the disease that is isolated to their skin (benign cutaneous malignant atrophic papulosis). However, identical skin findings can herald a lethal multiorgan variant (systemic malignant atrophic papulosis). The lack of involvement of internal organs over 3 years suggests that our patient has the benign cutaneous variant. Only time will tell whether systemic disease will develop.

More than 200 cases of malignant atrophic papulosis, including both the benign cutaneous form and the systemic form, have been published worldwide. ${ }^{1}$ However, the disease is likely underrecognized. Typically, malignant atrophic papulosis occurs in young white adults, often in their third decade of life. It has been described in patients of all ages and ethnic backgrounds. In 1997, Katz described a familial form of systemic malignant atrophic papulosis with a male-to-female ratio of about $3: 1 .^{2}$ The benign form is more commonly reported in women, with a female-to-male ratio of $3: 1 .^{3}$

The pathognomonic features in both the benign cutaneous and systemic forms of malignant atrophic papulosis are erythematous to pink papules of 2 to $15 \mathrm{~mm}$ in diameter that develop central

Box 1: Differential diagnosis of the cutaneous manifestations of malignant atrophic papulosis

- Atrophie blanche

- Guttate lichen sclerosus

- Guttate morphea

- Dermal mucinosis

- Systemic lupus erythematosus

- Dermatomyositis

- Antiphospholipid antibody syndrome

- Scleroderma

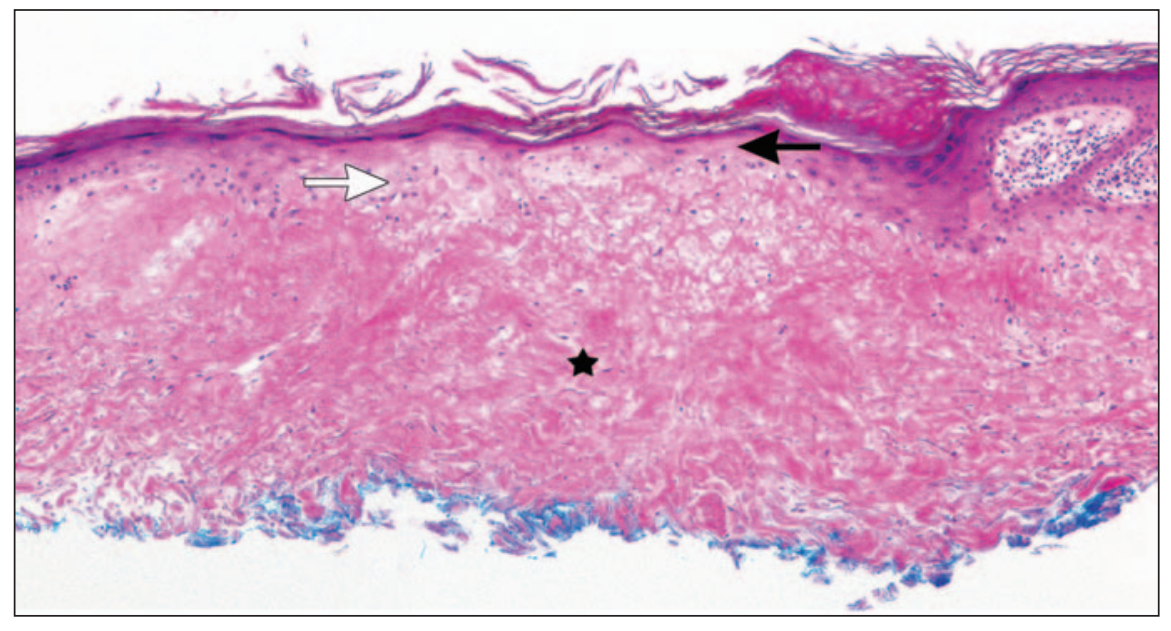

Figure 2: Histologic findings showing epidermal atrophy and squamatization (solid arrow), a vacuolar interface reaction (white arrow) and a wedge-shaped area of collagen degeneration and dermal sclerosis (star) (hematoxylin and eosin stain, original magnification $\times 40$ ).

porcelain-white, atrophic scars. The papules are often surrounded by a telangiectatic rim, may be dome-shaped and occasionally form a clover-like pattern. The average number of papules per patient is about 30. However, over 600 lesions have been described in a single patient. ${ }^{1}$ The proximal body surfaces, genitals and back are most often involved. The face, palms and soles are generally spared. Although the lesions are usually asymptomatic, some patients may experience a burning sensation.

Histopathologic evaluation of a skin biopsy is required to diagnose malignant atrophic papulosis and distinguish its lesions from clinical entities with similar presentations (Box 1). Characteristic histologic findings include a wedge-shaped area of collagen degeneration, an interface reaction with squamatization of the dermal-epidermal junction, a developing zone of papillary dermal sclerosis, melanin incontinence and epidermal atrophy (Figure 2). ${ }^{1}$ Early lesions have a perivascular, periadnexal and perineural inflammatory infiltrate with interstitial mucin deposition. ${ }^{1}$ Swelling and proliferation of endothelial cells and thrombosis may be seen beneath the zone of necrobiosis. ${ }^{1}$ Histologically, the lesions of the benign cutaneous variant and the systemic variant are indistinguishable.

Skin lesions are often the first and only sign heralding the onset of systemic malignant atrophic papulosis.
The systemic form is frequently fatal and typically develops within weeks to years after the onset of skin lesions. Involvement of internal organs rarely precedes the onset of cutaneous disease. The gastrointestinal, neurologic, ophthalmologic, cardiopulmonary and hepatorenal systems may be involved. However, intestinal perforation remains the leading cause of death. ${ }^{2}$ Fatal hemorrhagic or ischemic strokes, disabling polyradiculoneuropathy and nonspecific neurologic symptoms are among the neurologic complications of malignant atrophic papulosis. ${ }^{4}$ Ocular complications of the systemic variant include posterior subcapsular cataracts, diplopia, visual field defects, ptosis, oculomotor nerve palsies, blepharoptosis, optic atrophy and neuritis, papilledema and scleral plaques. ${ }^{5}$ Constrictive pericarditis, pleuritis, pleural effusions and death from myocardial infarctions have also been reported. ${ }^{1} \mathrm{Fi}-$ nally, vasculitis has been described in the hepatorenal systems. ${ }^{1}$ In patients with systemic malignant atrophic papulosis, death usually occurs within 2 3 years of involvement of internal organs. ${ }^{2}$ No specific laboratory tests for the disease are available, and there are no tests to distinguish between the benign and the systemic forms.

Because the systemic variant is frequently fatal, asymptomatic patients with skin disease often have symptoms of anxiety and depression. Reassurance 
that they have the benign cutaneous form comes if target organs are found not to be involved at baseline and over time. Nevertheless, the optimal methods and frequency of testing for systemic involvement remain unclear. Many treatments have been tried, yet few have consistently shown effectiveness in the management of malignant atrophic papulosis. Small case series suggest that antiplatelet agents (i.e., acetylsalicylic acid and dipyridamole) and intravenous immunoglobulin may prevent progression of the disease. ${ }^{1,6}$ In our patient, low-dose therapy with acetylsalicylic acid $(81 \mathrm{mg} / \mathrm{d})$ prevented the development of new skin lesions. We have found no evidence of systemic involvement during a followup period of 3 years.

\section{P. Régine Mydlarski MD}

Division of Dermatology

Department of Medicine University of Calgary

\section{Duane F. Barber MD}

Department of Pathology and

Laboratory Medicine

University of Calgary

Calgary Laboratory Services

Lynne H. Robertson MD

Division of Dermatology

Department of Medicine

University of Calgary

Calgary, Alta.

This article has been peer reviewed.

Competing interests: None declared.

\section{REFERENCES}

1. Scheinfeld N. Malignant atrophic papulosis. Clin Exp Dermatol 2007;32:483-7.

2. Katz SK, Mudd LJ, Roenigk HH Jr. Malignant atrophic papulosis (Degos' disease) involving three generations of a family. J Am Acad Dermatol 1997;37:480-4.

3. Wilson J, Walling HW, Stone MS. Benign cutaneous Degos disease in a 16-year-old girl. Pediatr Dermatol 2007;24:18-24.

4. Subbiah P, Wijdicks E, Muenter M, et al. Skin lesion with a fatal neurologic outcome (Degos'disease). Neurology 1996;46:636-40.
5. Egan R, Lessell S. Posterior subcapsular cataracts in Degos disease. Am J Ophthalmol 2000;129:806-7.

6. Zhu KJ, Zhou Q, Lin AH, et al. The use of intravenous immunoglobulin in cutaneous and recurrent perforating intestinal Degos disease (malignant atrophic papulosis). Br J Dermatol 2007;157:206-7.

CMAJ invites submissions to "What is your call?" Clinical details (including images) are presented on the first page along with a multiple-choice question about the diagnosis. The answer and a brief discussion of the condition follow on the second page. We specifically invite submissions illustrating common or important radiographic and electrocardiographic diagnoses of appeal to a general audience. We generally allow up to 5 references and require authors to obtain consent from the patient for publication of his or her story (form available at www.cmaj.ca/authors/checklist .shtml). Submit manuscripts online at http://mc.manuscriptcentral.com/cmaj.

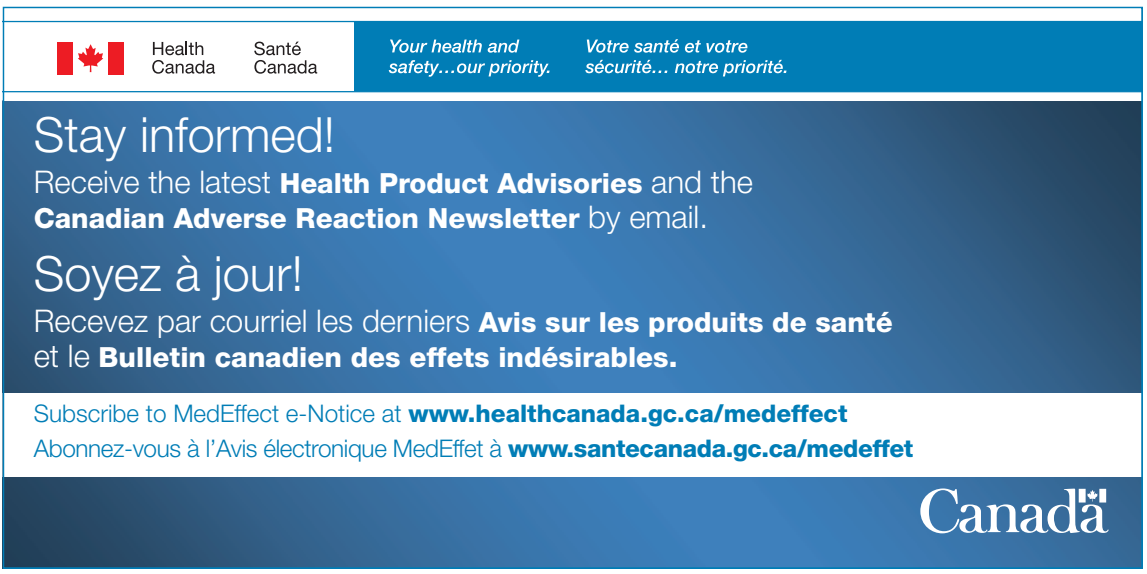

